\title{
The hadronic vacuum polarization of the muon from four-flavor lattice QCD
}

C. DeTar ${ }^{*} \stackrel{\dagger}{\text {, C.T.H. Davies }}{ }^{b}$, A.X. El-Khadra ${ }^{c f}$, E. Gámiz ${ }^{d}$, Steven Gottlieb ${ }^{e}$, D. Hatton ${ }^{b}$, A.S. Kronfeld ${ }^{f}$, J. Laiho ${ }^{g}$, G.P. Lepage ${ }^{h}$, Yuzhi Liu $^{d}$, P.B. Mackenzie ${ }^{f}$, C. McNeile ${ }^{i}$, E.T. Neili ${ }^{j}$, T. Primer ${ }^{k}$, J.N. Simone ${ }^{f}$, D. Toussaint ${ }^{k}$, R.S. Van de Water ${ }^{f}$, A. Vaquero $^{a}$, and Shuhei Yamamoto ${ }^{a}$

${ }^{a}$ Department of Physics and Astronomy, University of Utah, Salt Lake City, UT 84112 USA

${ }^{b}$ SUPA, School of Physics and Astronomy, University of Glasgow, Glasgow, G12 8QQ, UK

${ }^{c}$ Department of Physics, University of Illinois, Urbana, IL 61801, USA

${ }^{d}$ CAFPE and Departamento de Física Teórica y del Cosmos, Universidad de Granada, E-18071 Granada, Spain

${ }^{e}$ Department of Physics, Indiana University, Bloomington, IN 47405, USA

${ }^{f}$ Fermi National Accelerator Laboratory; Batavia, IL 60510 USA

${ }^{g}$ Department of Physics, Syracuse University, Syracuse, NY 13244, USA

${ }^{h}$ Laboratory for Elementary-Particle Physics, Cornell University, Ithaca, New York 14853, USA

${ }^{i}$ Centre for Mathematical Sciences, University of Plymouth, PLA 8AA, UK

${ }^{j}$ Department of Physics, University of Colorado, Boulder, CO 80309, USA

${ }^{k}$ Physics Department, University of Arizona, Tucson, AZ 85721, USA

\section{Fermilab Lattice, HPQCD, and MILC Collaborations}

\begin{abstract}
We present an update on the ongoing calculations by the Fermilab Lattice, HPQCD, and MILC Collaboration of the leading-order (in electromagnetism) hadronic vacuum polarization contribution to the anomalous magnetic moment of the muon. Our project employs ensembles with four flavors of highly improved staggered fermions, physical light-quark masses, and four lattice spacings ranging from $a \approx 0.06$ to $0.15 \mathrm{fm}$ for most of the results thus far.
\end{abstract}

37th International Symposium on Lattice Field Theory - Lattice2019

16-22 June 2019

Wuhan, China

\footnotetext{
* Speaker.

†email: detarephysics.utah.edu

$\ddagger$ Operated by Fermi Research Alliance, LLC, under Contract No. DE-AC02-07CH11359 with the US DOE.
} 


\section{Introduction}

The measured anomalous magnetic moment of the muon $a_{\mu}=\left(g_{\mu}-2\right) / 2$ disagrees with current standard-model predictions by more than three standard deviations $[1,2,3]$. This discrepancy has generated considerable interest as a possible sign of new and as-yet-undiscovered fundamental processes. A concerted effort is now underway to reduce both theoretical and experimental uncertainties to clarify the disagreement. Improved experiments at Fermilab (E989) and planned at J-PARC (E34) aim to reduce the measured uncertainty by a factor of about four. To match this on the theoretical side requires an uncertainty of approximately $0.3 \%$ in the predicted hadronic contribution, which is the largest source of theoretical uncertainty. The most precise theoretical prediction for this contribution uses dispersion relations together with the measured cross section for $e^{+} e^{-}$annihilation to hadrons through a single virtual photon. The uncertainties in that method become difficult to control at the level of precision now sought. Because it is an ab initio method, lattice QCD (plus QED) can provide the needed small uncertainty, but present calculations by several groups are still quite far from this goal. Here, we describe progress in the effort of our collaboration towards reducing the lattice QCD errors to the target uncertainty. This report updates Ref. [4] and includes new, preliminary results for the quark-line disconnected contribution.

\section{Formalism}

We use both the time-moment method and time-momentum method [5], which start from a lattice calculation of the electromagnetic current-density correlator at zero momentum:

$$
G(t)=\frac{1}{3} \int d \mathbf{x} \sum_{f} q_{f}^{2} Z_{V}^{2}\left\langle j_{i}^{f}(\mathbf{x}, t) j_{i}^{f}(\mathbf{0}, 0)\right\rangle
$$

where the sum is over flavors $f$ with charges $q_{f}$. In the continuum, the reduced vacuum polarization

$$
\hat{\Pi}\left(\omega^{2}\right)=\frac{4 \pi^{2}}{\omega^{2}} \int_{0}^{\infty} d t G(t)\left[\omega^{2} t^{2}-4 \sin ^{2}\left(\frac{\omega t}{2}\right)\right]
$$

determines the leading-order hadronic contribution to anomalous magnetic moment

$$
a_{\mu}^{\mathrm{HVP}, \mathrm{LO}}=\left(\frac{\alpha}{\pi}\right)^{2} \int_{0}^{\infty} d Q^{2} K_{E}\left(Q^{2}\right) \hat{\Pi}\left(Q^{2}\right),
$$

where $K_{E}\left(Q^{2}\right)$ is the Schwinger-term loop integrand as formulated by Blum [6]. The time-moment method expands the reduced vacuum polarization in powers of $Q^{2}$

$$
\hat{\Pi}\left(Q^{2}\right)=\sum_{j=1}^{\infty} Q^{2 j} \Pi_{j}
$$

where, on the lattice, the Taylor coefficients are determined by the discrete time-moments of $G(t)$, i.e., $q_{f}^{2} G_{2 n}=(-)^{n}(2 n) ! \Pi_{n-1}$, where

$$
G_{2 n} \equiv a^{4} \sum_{t, \mathbf{x}, f} t^{2 n} q_{f}^{2} Z_{V}^{2}\left\langle j_{i}^{f}(\mathbf{x}, t) j_{i}^{f}(\mathbf{0}, 0)\right\rangle .
$$

A Padé approximant is then used to extend the value of $\hat{\Pi}\left(Q^{2}\right)$ to small $Q^{2}$.

Corrections for finite-volume and discretization effects are applied to the Taylor coefficients $\Pi_{n}$ using a chiral model before inserting into the integral in Eq. (2.3). 

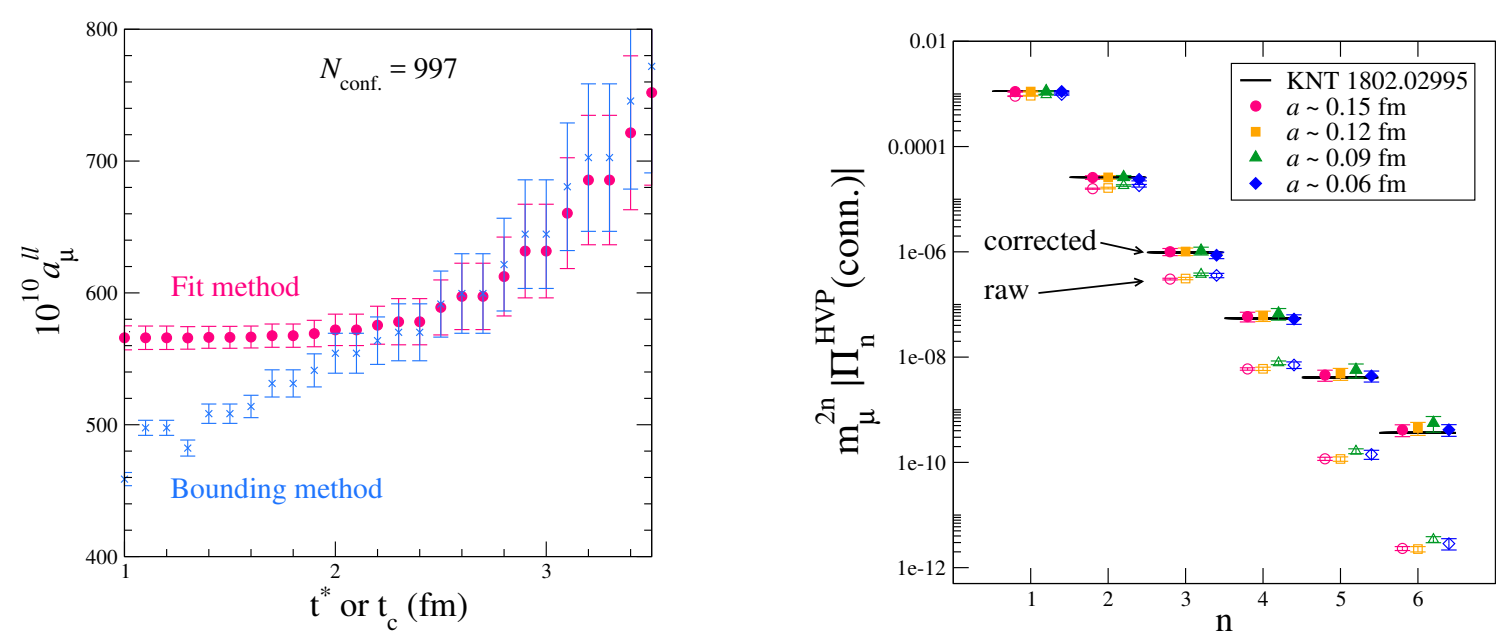

Figure 1: Left: Values of $a_{\mu}^{l l}$ obtained with different methods for handling the high $t$ contribution, namely, our "fit method" and the BMW "bounding method" [10]. They agree well for $t>2.3 \mathrm{fm}$. Right: Raw (open symbols) and corrected (filled symbols) vacuum-polarization Taylor coefficients. They agree well with results from the R-ratio method, shown with black lines [3].

\section{Results}

\subsection{Light quark-line-connected contribution}

Our calculations are based on gauge-field ensembles at four lattice spacings, namely, approximately $0.15,0.12,0.09$, and $0.06 \mathrm{fm}$. These ensembles were generated in the presence of $(2+1+1)-$ flavors of highly-improved staggered sea quarks (HISQ) [8] with masses close to their physical values [9]. Measurements are done with the same physical-mass quarks.

The vector-current-density correlator in Eq. 2.1 separates into a larger quark-line-connected and a smaller, but more difficult to calculate, quark-line-disconnected contributions. We first discuss the large connected light-quark contribution (with degenerate up and down quarks). The largest source of statistical uncertainty comes from the rapidly growing noise-to-signal ratio in the current-density correlator at large Euclidean time $t$. There are a variety of strategies for treating it. We fit the vector-current-density correlator to a set of exponentials and, for $t$ larger than a cutoff $t^{*}$, replace the lattice values by an extrapolation of the fit. We show in Fig. 1 (left) that for $t^{*}>2.3 \mathrm{fm}$ the result for the contribution to the anomalous magnetic moment agrees with the BMW bounding method [10]. One might be concerned that the extrapolation is risky because our fit model does not include the full spectrum of states. We are able to show with a chiral model that the fit method with only two intermediate states is, in fact, reliable at the level of accuracy we need [4].

The lattice results require corrections for finite-size and discretization effects. A large part of these corrections are estimated through the use of a chiral model. The corrected Taylor coefficients $\Pi_{n}$ are shown in Fig. 1. It is noteworthy that our lattice result agrees with the phenomenological result based on R-ratios [3] at the $1-2 \%$ level. The corrected values of $a_{\mu}^{l l}$ are then extrapolated to zero lattice spacing, as shown in Fig. 2, with the result $a_{\mu}^{\ell \ell}=637.8(8.8) \times 10^{-10}$, i.e., with an error of $1.4 \%{ }^{1}$ The error breakdown is shown in Table 1 . The dominant contributions to the total

\footnotetext{
${ }^{1}$ This result has been updated since the conference.
} 
Table 1: Error budget for the quark-line connected light-quark contribution

\begin{tabular}{ll}
\hline Lattice spacing uncertainty $\left(w_{0}\right)$ & $0.8 \%$ \\
Monte Carlo statistics & 0.7 \\
Continuum extrapolation & 0.7 \\
Finite volume and discretization corrections & 0.6 \\
Current renormalization & 0.1 \\
Chiral interpolation & 0.1 \\
Strange sea quark mass adjustment & 0.1 \\
\hline Total & $1.4 \%$ \\
\hline
\end{tabular}
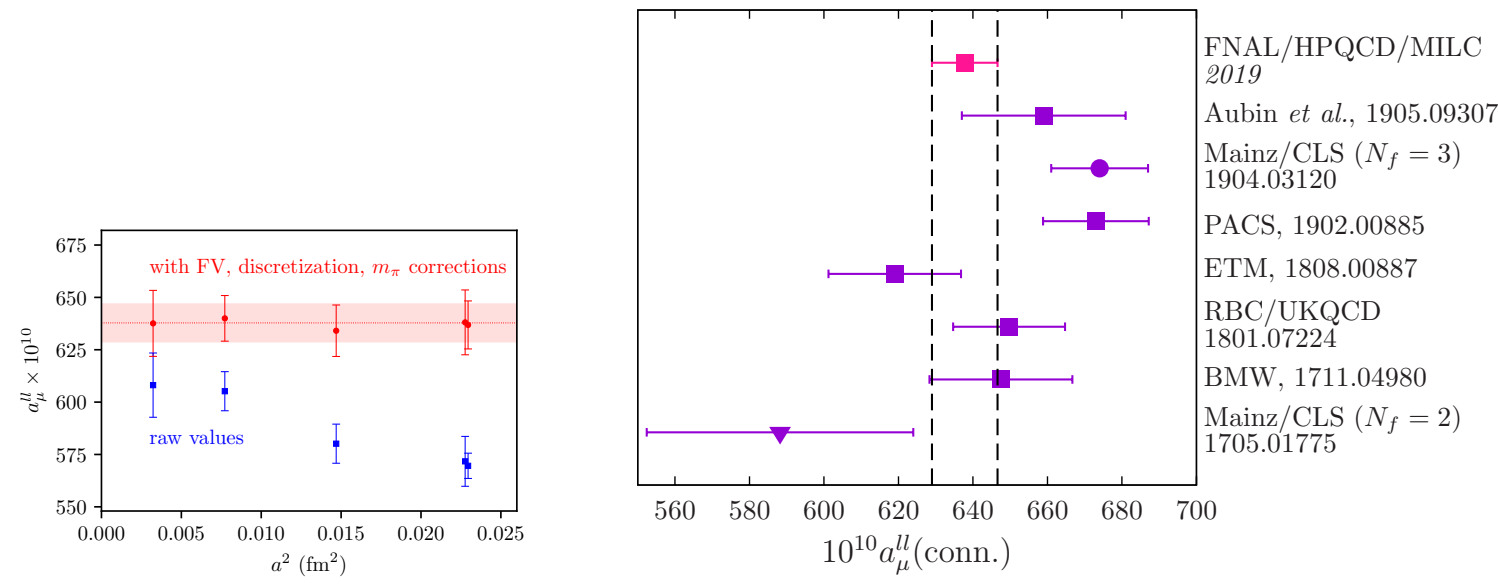

Figure 2: Left: Continuum extrapolation of our results for the light-quark contribution $a_{\mu}^{l l}$. Shown are values before (open blue symbols) and after (filled red symbols) applying corrections for finite-volume and discretization effects. Right: Comparison of our result for the total light-quark contribution with those of other groups $[11,12,13,14,15,7,16]$.

error on $a_{\mu}^{\ell} \ell$ are the lattice spacing uncertainty, statistics, the continuum extrapolation, and finite volume and discretization corrections. Our result is compared with recent results of other groups [11, 12, 13, 14, 15, 7, 16] in Fig. 2.

\subsection{Total leading-order light-quark hadron vacuum polarization}

To obtain the total leading-order up/down quark contribution to $a_{\mu}$ we need to include strong isospin-breaking and electromagnetic effects, and we need the quark-line-disconnected contribution. A large part of the strong isospin-breaking comes from the splitting of the pion multiplet in the two-pion intermediate state. This contribution can be calculated from the same chiral model used in the finite-volume and lattice-discretization correction. The same procedure can also be used to estimate the quark-line-disconnected contribution. (Our more recent, explicit calculation, discussed below, was not used in estimating this contribution.) The residual (non-pion-pion) contributions are then estimated conservatively, as shown in Table 2. In the case of strong isospin breaking, explicit lattice calculations help provide the estimates shown $[15,17,18]$. The largest uncertainty here 
Table 2: Adjustments to obtain the total up/down quark contribution to $a_{\mu}^{u d}$. All values are in units of $10^{-10}$.

\begin{tabular}{lr}
\hline$M\left(\pi^{0}\right)-M\left(\pi^{+}\right)$ & -4.3 \\
$\pi-\pi$ disconnected & -7.9 \\
\hline Total & $-12(3)$ \\
\hline disconnected & $-5(5)$ \\
Strong isospin breaking & $10(10)$ \\
Electromagnetism & $0(5)$ \\
\hline Total adjustment & $-7(13)$ \\
\hline
\end{tabular}

still comes from strong isospin breaking. Altogether, then, the total up-down quark contribution is, then, $a_{\mu}^{u d}=630.8(8.8)(13) \times 10^{-10}$, where the first error comes from the light-quark value above and the second from the corrections in Table 2.

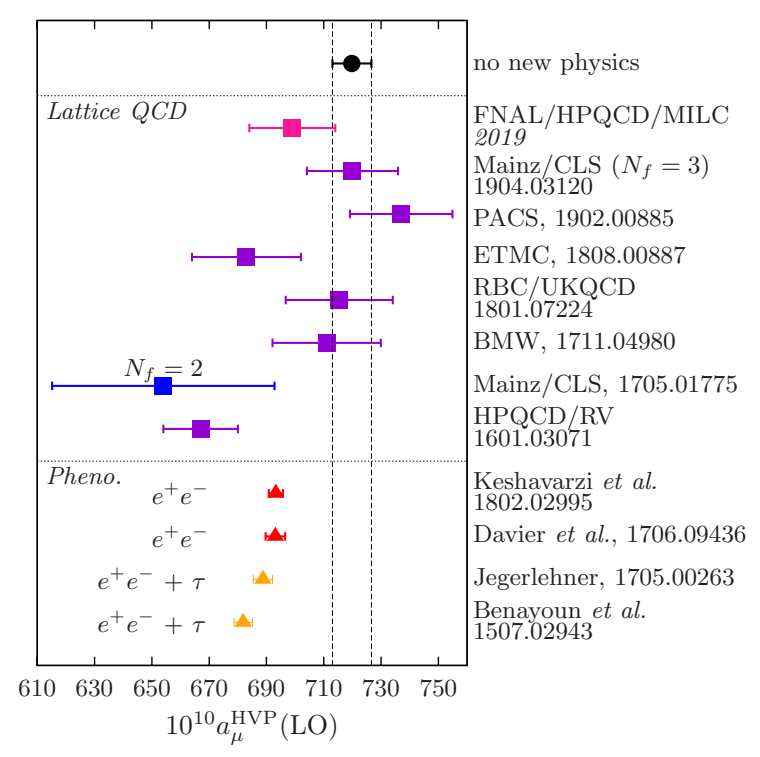

Figure 3: Comparison of results for the total leading-order hadronic vacuum polarization contribution to $a_{\mu}^{\mathrm{LO}, \mathrm{HVP}}[1,2,3,12,13,14,15,7,16,19,20]$.

Finally, including further contributions from the strange, charm, and bottom quarks [5, 21, 22] gives $a_{\mathrm{HVP}-\mathrm{LO}}^{\mu}=699(15)_{u, d}(1)_{s, c, b} \times 10^{-10}$, where the errors are from the light-quark and heavierquark contributions, respectively. The largest contribution to the uncertainty comes from isospinbreaking and electromagnetic corrections. This value is compared with those of others in Fig. 3.

\section{Quark-line-disconnected HVP}

We conclude by presenting some preliminary results from our explicit lattice calculation of the quark-line-disconnected contribution, which will eventually replace the model estimates described 


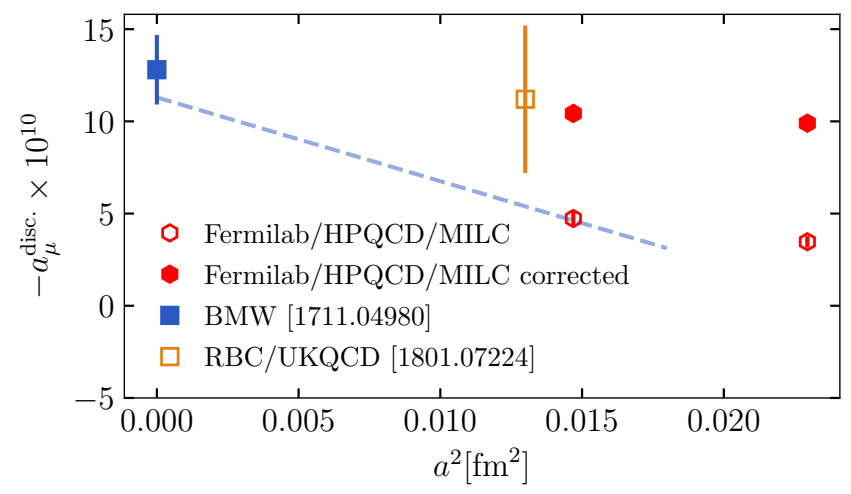

Figure 4: Our preliminary results for the quark-line disconnected contribution to $a_{\mu}^{\mathrm{LO}, \mathrm{HVP}}$, compared with those of the BMW [7] and RBC/UKQCD [15] collaborations. Our results are shown before and after applying a correction for finite-volume and discretization effects.

above. So far we have results for simulations at only two lattice spacings 0.15 and $0.12 \mathrm{fm}$, updated from work described in Ref. [23]. The resulting contributions are summarized in Fig. 4 and compared with results from the BMW [7] and RBC/UKQCD Collaborations [15]. The results are corrected for finite-volume and discretization effects, which removes most of the lattice-spacing dependence. We see that the finite-volume plus discretization-error correction is significant. Although the extrapolated value is statistically consistent with the model estimates described above, a calculation at a finer lattice spacing is evidently needed in order to meet our goals for a reduction in the overall error.

\section{Outlook}

We continue in our efforts to reduce the lattice uncertainty. Besides increasing statistics and working at finer lattice spacing, we are also working to reduce the uncertainties in the lattice scale, we are planning calculations of electromagnetic corrections, and we are investigating lattice calculations of contributions from two-hadron intermediate states.

\section{Acknowledgments}

This work is supported by grants from the US Department of Energy, the US National Science Foundation, the United Kingdom STFC, MINECO (Spain), the Junta de Andalucía (Spain), the German Excellence Initiative and the European Union Seventh Framework Program, the European Union's Marie Curie COFUND program, and the Blue Waters PAID program. Computations were carried out on USQCD LQCD clusters and at the US DOE NERSC and ALCF centers, the U.K. STFC DiRAC HPC Facility, the US NSF TACC XSEDE center, and computers at the University of Colorado and Indiana University.

\section{References}

[1] F. Jegerlehner, EPJ Web Conf. 166, 00022 (2018) doi:10.1051/epjconf/201816600022 [arXiv:1705.00263 [hep-ph]]. 
[2] M. Davier, A. Hoecker, B. Malaescu and Z. Zhang, Eur. Phys. J. C 77, 827 (2017) doi:10.1140/epjc/s10052-017-5161-6 [arXiv:1706.09436 [hep-ph]].

[3] A. Keshavarzi, D. Nomura and T. Teubner, Phys. Rev. D 97, 114025 (2018) doi:10.1103/PhysRevD.97.114025 [arXiv:1802.02995 [hep-ph]] and private communication.

[4] C. T. H. Davies et al. [Fermilab Lattice, HPQCD, and MILC Collaborations], arXiv:1902.04223.

[5] B. Chakraborty et al. [HPQCD Collaboration], Phys. Rev. D 89, 114501 (2014) doi:10.1103/PhysRevD.89.114501 [arXiv:1403.1778 [hep-lat]].

[6] T. Blum, Phys. Rev. Lett. 91, 052001 (2003) doi:10.1103/PhysRevLett.91.052001 [hep-lat/0212018].

[7] S. Borsanyi et al. [Budapest-Marseille-Wuppertal Collaboration], Phys. Rev. Lett. 121, 022002 (2018) doi:10.1103/PhysRevLett.121.022002 [arXiv:1711.04980 [hep-lat]].

[8] E. Follana et al. [HPQCD and UKQCD Collaborations], Phys. Rev. D 75, 054502 (2007) doi:10.1103/PhysRevD.75.054502 [hep-lat/0610092].

[9] A. Bazavov et al. [MILC Collaboration], Phys. Rev. D 87054505 (2013) doi:10.1103/PhysRevD.87.054505 [arXiv:1212.4768 [hep-lat]].

[10] S. Borsanyi et al., Phys. Rev. D 96074507 (2017) doi:10.1103/PhysRevD.96.074507 [arXiv:1612.02364 [hep-lat]].

[11] C. Aubin, T. Blum, C. Tu, M. Golterman, C. Jung and S. Peris, arXiv:1905.09307 [hep-lat].

[12] A. Gérardin et al., Phys. Rev. D 100, 014510 (2019) doi:10.1103/PhysRevD.100.014510 [arXiv:1904.03120 [hep-lat]].

[13] E. Shintani et al. [PACS Collaboration], Phys. Rev. D 100034517 (2019) doi:10.1103/PhysRevD.100.034517 [arXiv:1902.00885 [hep-lat]].

[14] D. Giusti, F. Sanfilippo and S. Simula, Phys. Rev. D 98114504 (2018) doi:10.1103/PhysRevD.98.114504 [arXiv:1808.00887 [hep-lat]].

[15] T. Blum et al. [RBC and UKQCD Collaborations], Phys. Rev. Lett. 121, 022003 (2018) doi:10.1103/PhysRevLett.121.022003 [arXiv:1801.07224 [hep-lat]].

[16] M. Della Morte et al., JHEP 1710, 020 (2017) doi:10.1007/JHEP10(2017)020 [arXiv:1705.01775].

[17] B. Chakraborty et al. [Fermilab Lattice,HPQCD, and MILC Collaborations], Phys. Rev. Lett. 120. 15, 152001 (2018) doi:10.1103/PhysRevLett.120.152001 [arXiv:1710.11212 [hep-lat]].

[18] D. Giusti, V. Lubicz, G. Martinelli, F. Sanfilippo and S. Simula, Phys. Rev. D 99 11, 114502 (2019) doi:10.1103/PhysRevD.99.114502 [arXiv:1901.10462 [hep-lat]].

[19] B. Chakraborty, C. T. H. Davies, P. G. de Oliviera, J. Koponen, G. P. Lepage and R. S. Van de Water, Phys. Rev. D 96034516 (2017) doi:10.1103/PhysRevD.96.034516 [arXiv:1601.03071 [hep-lat]].

[20] M. Benayoun, P. David, L. DelBuono and F. Jegerlehner, Eur. Phys. J. C 75, 613 (2015) doi:10.1140/epjc/s10052-015-3830-x [arXiv:1507.02943 [hep-ph]].

[21] G. C. Donald, C. T. H. Davies, R. J. Dowdall, E. Follana, K. Hornbostel, J. Koponen, G. P. Lepage and C. McNeile, Phys. Rev. D 86, 094501 (2012) doi:10.1103/PhysRevD.86.094501 [arXiv:1208.2855 [hep-lat]].

[22] B. Colquhoun, R. J. Dowdall, C. T. H. Davies, K. Hornbostel and G. P. Lepage, Phys. Rev. D 91, 074514 (2015) doi:10.1103/PhysRevD.91.074514 [arXiv:1408.5768 [hep-lat]].

[23] S. Yamamoto et al. [Fermilab Lattice, HPQCD, and MILC Collaborations], PoS LATTICE 2018, 322 (2019) doi:10.22323/1.334.0322 [arXiv:1811.06058 [hep-lat]]. 